\title{
DECIDABLE VARIETIES WITH MODULAR CONGRUENCE LATTICES
}

\author{
BY S. BURRIS ${ }^{1}$ AND R. MCKENZIE ${ }^{2}$
}

\begin{abstract}
For a large collection of varieties we show that if the first-order theory of such a variety is decidable then the variety decomposes into the product of two well-known highly specialized varieties. For many varieties the decidability question then reduces to a decidability question about modules.
\end{abstract}

A variety is a class of (abstract) algebras (belonging to some language) closed under the formation of direct products, subalgebras and homomorphic images. A variety $V$ is locally finite if every finitely-generated member of $V$ is finite. A class of algebras $K$ generates a variety $V$ if $V$ is the smallest variety containing $K$-then we say that $V$ is generated by $K$, written $V=V(K)$. A variety is finitely generated if it is generated by finitely many finite algebras, or equivalently by a single finite algebra. The kernel of a homomorphism is called a congruence, and the congruences of any algebra form a lattice. A variety is congruence modular, or we prefer to say just modular, if the lattice of congruences of every algebra in the variety satisfies the modular law. (Most of the well-studied varieties are modular; for example varieties of groups, rings, modules and lattices are modular. However, the variety of semigroups is not modular.)

A variety $V$ is a product of two subvarieties $V_{1}, V_{2}$ if $V_{1} \cup V_{2}$ generates $V$ and there is a term $b(x, y)$ such that $V_{1} \vDash b(x, y)=x, V_{2} \vDash b(x, y)=y$. If this is so we write $V=V_{1} \otimes V_{2}$, and then for every algebra $A$ in $V$ there are (up to isomorphism) unique algebras $A_{1} \in V_{1}, A_{2} \in V_{2}$ such that $A \cong A_{1} \times A_{2}$. A class $K$ of first-order structures has a decidable theory if there is an effective procedure to determine precisely which first-order sentences are true of every member of $K$.

A variety $U$ is a discriminator variety if it is generated by a set $K$ for which there exists a ternary term $t(x, y, z)$ such that $K$ satisfies $t(x, y, z)=x$ if $x \neq y$; $=z$ if $x=y$. In everyday mathematics such varieties appear only as highly specialized varieties of rings, or varieties associated with algebraic logics.

Received by the editors December 29, 1980.

1980 Mathematics Subject Classification. Primary 03B25, 08B10, 08 A05.

Key words and phrases. Decidable theory, modular variety, discriminator variety, abelian variety, product of varieties, unitary left $R$-modules.

1 Research supported by NSERC Grant No. A7256.

2 Research supported by NSF Grant No. MCS 77-22913.

() 1981 American Mathematical Society $0002-9904 / 81 / 0000-0210 / \$ 01.75$ 
The center $Z_{A}$ of an algebra $A$ is the binary relation defined by

$$
(a, b) \in Z_{A} \text { iff } \forall t \forall \tilde{u} \forall \widetilde{v}[t(\widetilde{u}, a)=t(\widetilde{v}, a) \leftrightarrow t(\widetilde{u}, b)=t(\widetilde{v}, b)],
$$

where $t$ denotes an $m+1$-ary term and $\tilde{u}, \tilde{v}$ are $m$-ary sequences of variables, for any $m<\omega . Z_{A}$ is actually a congruence on $A$. An algebra is abelian if $V(A)$ is modular and $Z_{A}=A \times A$. Given any modular variety $V$ the abelian algebras in $V$ form a subvariety $V_{a b}$.

Let $B P^{1}$ denote the class of structures $\left(B, B_{0}, \vee, \wedge,{ }^{\prime}, 0,1\right)$ where $\left(B, \vee, \wedge,{ }^{\prime}, 0,1\right)$ is an atomic Boolean algebra and $\left(B_{0}, \vee, \wedge, ', 0,1\right)$ is a subalgebra containing all the atoms of $B$.

THEOREM 1. The theory of $B P^{1}$ is undecidable.

Proof. The class of finite graphs can be interpreted in $B P^{1}$ along the lines of a construction introduced by M. Rubin [6].

THEOREM 2. If $V$ is a locally finite modular variety such that every reduct of $V$ to a finite language has a decidable theory, then $V$ has a subvariety $V_{d s}$ which is a discriminator variety and $V=V_{d s} \otimes V_{a b}$.

Proof. We focus our attention on three special kinds of finite algebras.

(Type I) $A$ is subdirectly irreducible and there is a subalgebra $B$ of $A$ and a congruence $\theta$ of $B$ such that $Z_{A} \cap(B \times B)<\theta<B \times B$.

(Type II) $A$ is subdirectly irreducible nonabelian with $Z_{A}=0$, and there is an abelian subalgebra $B$ of $A$ with $|B|>1$.

(Type III) $A$ is directly indecomposable but not simple and $V(A)$ is congruence distributive (i.e. every algebra in $V(A)$ has a lattice of congruences which satisfies the distributive law).

If $V$ contains an algebra $A$ of Type I or III then, using a modification of the Boolean power construction, we can interpret $B P^{1}$ into the class of subdirect powers of $A$. If $V$ contains an algebra $A$ of Type II then, extending a technique pioneered by Zamjatin [8] for the study of groups, finite bipartite graphs can be interpreted into the class of subdirect powers of $A$.

It follows that no finite member of $V$ can be of Type I, II or III. Then using A. Pixley's characterization [5] of finitely-generated discriminator varieties plus the modular commutator introduced by J. Hagemann, C. Herrmann and J. D. H. Smith [3] , [4] , [7] and two results of R. Freese and R. McKenzie [2], [2a], one can prove that $V$ has the desired decomposition.

THEOREM 3. The question of 'which modular varieties $V(A)$, generated by a finite algebra $A$ belonging to a finite language, have a decidable theory' effectively reduces to the question of 'which finite rings $R$ are such that the class of unitary left R-modules has a decidable theory'. 
Proof. Given a finite algebra $A$ of finite type one can effectively determine if $V(A)$ is modular, and also if $V(A)$ is of the form $V_{d s} \otimes V_{a b}$ where $V_{d s}$ is a discriminator variety and $V_{a b}$ is abelian. H. Werner (see [1]), extending results of $\mathrm{S}$. Comer, proved that every finitely-generated discriminator variety belonging to a finite language has a decidable theory. If $V(A)=V_{d s} \otimes V_{a b}$ then one can effectively construct a finite ring $R$, given $A$, such that a decision procedure for the theory of $V_{a b}$ yields a decision procedure for the theory of unitary left $R$-modules, and vice-versa.

\section{REFERENCES}

1. S. Burris and H. Werner, Sheaf constructions and their elementary properties, Trans. Amer. Math. Soc. 248 (1979), 269-309.

2. R. Freese and R. McKenzie, The commutator, an overview (preprint).

2a. R. Freese and R. McKenzie, Residually small varieties with modular congruence lattices, Trans. Amer. Math. Soc. 264 (1981), 419-430.

3. J. Hagemann and C. Herrmann, A concrete ideal multiplication for algebraic systems and its relation to congruence distributivity, Arch. Math. (Basel) 32 (1979), 234-245.

4. C. Herrmann, Affine algebras in congruence modular varieties, Acta Sci. Math. (Szeged) 41 (1979), 119-125.

5. A. F. Pixley, Completeness in arithmetical algebras, Algebra Universalis 2 (1972), 179-196.

6. M. Rubin, The theory of Boolean algebras with a distinguished subalgebra is undecidable, Ann. Sci. Univ. Clermont No. 60 Math. No. 13 (1976), 129-134.

7. J. D. H. Smith, Mal'cev varieties, Lecture Notes in Math., vol. 554, Springer-Verlag, Berlin and New York, 1976.

8. A. P. Zamjatin, A nonabelian variety of groups has an undecidable elementary theory, Algebra and Logic 17 (1978), 13-17.

\footnotetext{
DEPARTMENT OF PURE MATHEMATICS, UNIVERSITY OF WATERLOO, WATERLOO, ONTARIO, CANADA N2L 3G1

DEPARTMENT OF MATHEMATICS, UNIVERSITY OF CALIFORNIA, BERKELEY, BERKELEY, CALIFORNIA 94720
} 